\title{
Bending of functionally graded plates via a refined quasi-3D shear and normal deformation theory
}

https://doi.org/10.1515/cls-2018-0014

Received Apr 08, 2018; accepted Apr 17, 2018

\begin{abstract}
Bending of functionally graded plate with two reverse simply supported edges is studied based upon a refined quasi three-dimensional (quasi-3D) shear and normal deformation theory using a third-order shape function. The present theory accounts for the distribution of transvers shear stresses that satisfies the free transverse shear stresses condition on the upper and lower surfaces of the plate. Therefore, the strain distribution does not include the unwanted influences of transverse shear correction factor. The effect of transverse normal strain is included. Unlike the traditional normal and shear deformation theories, the present theory have four unknowns only. The equilibrium equations are derived by using the principle of virtual work. The influence of material properties, aspect and side-to-thickness ratios, mechanical loads and inhomogeneity parameter are discussed. The efficiency and correctness of the present theory results are established by comparisons with available theories results.
\end{abstract}

Keywords: FG Plate; bending; refined quasi-3D shear and normal theory; Navier's solution

\section{Introduction}

Functionally graded materials (FGMs) are new composite materials type, often composed of mixture of metals and ceramics. These materials are microscopically inhomogeneous, vary continuously in material characteristics from

\footnotetext{
${ }^{\star}$ Corresponding Author: Asharf M. Zenkour: Department of Mathematics, Faculty of Science, King Abdulaziz University, P.O. Box 80203, Jeddah 21589, Saudi Arabia;

Email: zenkour@gmail.com; Department of Mathematics, Faculty of Science, Kafrelsheikh University, Kafrelsheikh 33516, Egypt; Email: zenkour@sci.kfs.edu.eg

Rabab A. Alghanmi: Department of Mathematics, Faculty of Science, King Abdulaziz University, P.O. Box 80203, Jeddah 21589, Saudi Arabia; Department of Mathematics, Rabigh College for Sciences and Arts, King Abdulaziz University, P.O. Box 344, Jeddah 21911, Saudi Arabia; Email: raalghanmi@kau.edu.sa
}

one surface to other. This done by varying the combination of these materials gradually across the thickness to get smooth variations of material characteristics and best responses to the applied mechanical loading. FGMs are extensively used in numerous applications like nuclear, spacecraft, civil engineering, mechanics, automotive and power generation industries. The idea of FGMs appeared in 1984 in Japan [1].

The first-order shear deformation plate theory (FSDT) that needs a correction for the shear factor to achieve the transverse shear stress free conditions at the upper and lower surfaces of the considered plate has been introduced by Zhao and Liew [2] and Hosseini-Hashemi et al. [3]. Solution for the bending of FG plates by four-variable plate theory was given by Thai and Choi [4]. Cheng and Batra [5] have applied a finite element method to verify the accuracy of the classical plate theory (CPT). Carrera et al. [6] have used Carrera's Unified Formulation for analyzing FG plates and shells. Exact solution of the simply supported FG plates was obtained by Vel and Batra [7]. Reddy [8] has used third-order shear deformation theory (TSDT) to study the FG plates. Zenkour [9] has developed a unified formula for FG sandwich plates that consist of three layers by applying sinusoidal shear deformation theory. A simple shear and normal deformation theory of higher-order rank for FG plates has been presented by Bouazza et al. [10]. Thai and Choi [11] have used a refined FSDT with four unknowns instead of five for FG plates. A Levy-type model for cylindrical bending FG plates has been used by Navazi et al. [12]. Rezaei Mojdehi et al. [13] have used Meshless local Petrov-Galerkin model for analyzing thick FG plates. Kim and Reddy [14] applied a TSDT for analyzing FG plates. Liu and Zhong [15] presented a 3D bending analysis of FG plate with a distributed elastic modulus. Matsunaga [16] introduced a theory of higher-order rank for the valuation of stresses in FG plates under mechanical and thermal loadings. Zhang et al. [17] have obtained a 3D solution for the bending of thick FG plates. Swaminathan et al. [18] have presented a survey of methods and theorems that used for studying FG plates. Mantari and Soares [19] have applied a recently developed higher-order shear deformation theory to study the bending of FG plates. Benachour et al. [20]

Ә Open Access. (c) 2018 A. M. Zenkour and R. A. Alghanmi, published by De Gruyter. 
have introduced a refined plate theory with four variables to analyze FG plates. A simple sinusoidal shear and normal deformations theory for the bending of FG plates has been introduced by Zenkour [21]. Carrera et al. [22] have estimated the thickness stretching influence on FG plate and shell. Neves et al. [23] have introduced sinusoidal shear deformation theory for analyzing FG by applying radial basis function collocation method. A simple four-unknown plate theory with four unknowns for analyzing the bending of FG plates has been proposed by Zenkour [24]. Zenkour [25] has used a generalized shear deformation theory to study the bending of FG undergo transverse uniform and sinusoidal loads. Dongdong et al. [26] have developed refined plate theory to investigate FG sandwich plates under the influence of thermal and mechanical loads. Akavci [27] has studied the mechanical response of FG sandwich plates on elastic foundation. Zenkour et al. [28] have studied the hygrothermal and mechanical effects on FG plates resting on elastic foundations. Zenkour and Radwan [29] have investigated the free vibration analysis of FG sandwich plates resting on Winkler-Pasternak foundations. A hyperbolic theory of the bending of FG plates resting on elastic foundation has been applied by Zenkour and Radwan [30]. Sandwich plates with FG face sheets and FG core have been presented by Dongdong et al. [31]. Rouzegar and Abbasi [32] have used four-variable refined theory and refined finite element model for investigating FG plates.

The quasi-3D shear deformation theories are higherorder shear deformation theories that consider the thickness stretching $\varepsilon_{z}$ by defining the out-of-plane displacement as a function in the thickness direction. Neves et al. [33] and [34] have introduced the in-plane displacements as a hyperbolic sine/ sinusoidal form in the thickness direction while the out-of-plane displacement is expressed in a quadratic polynomial form. Talha and Singh [35], and Reddy [36], have used quasi-3D shear deformation theory where the in-plane displacements are expressed in cubic variation and quadratic variation for the out-ofplane displacement. Generalized hybrid quasi-3D shear deformation theory with six unknowns has been used by Mantari and Guedes Soares [37]. Simple form for quasi3D shear deformation theory with sinusoidal variation for displacements has been improved by Thai and Kim [38] to analyze FG plates. Mantari and Guedes Soares [39, 40] have used hyperbolic/ tangential function for investigating graded plates with exponential form and graded plates with power law form. Akavci and Tanrikulu [41] have investigated bending and vibration of FG plates by applying new quasi-3D and 2D shear deformation theories.

\section{Formulation of the problem}

\subsection{FG plates}

Consider a functionally graded (FG) plate with thickness $h$, length $a$, width $b$ (Figure 1). An orthogonal axes, $x, y$, $z$, is selected, where the mid-surface of the plate defined by $z=0$. The effective properties $P$ of the FG layer namely, Young's modulus $E$ and Poisson's ratio $v$ are continuously varied in $z$ direction according to the formula (Bao and Wang [42])

$$
P(z)=P_{m}+\left(P_{c}-P_{m}\right)\left(\frac{1}{2}+\frac{z}{h}\right)^{k}, \quad k \geqslant 0,
$$

where $P_{m}$ and $P_{c}$ are metal and ceramic properties, respectively, and $k$ is the volume fraction exponent. The plate is completely ceramic when k equals to zero and completely metal when $k$ equals to infinity. The variation of the plate thickness depends on the exponent of the power law formula so the lower surface of FG plate $(z=-h / 2)$ is totally metal and the upper surface $(z=h / 2)$ is totally ceramic whereas the materials have different distributions among the upper and lower surfaces. It is to be noted that also different formulas could be used for the effective properties and volume fractions of the FG plate. Mori and Tanaka [43] have presented their scheme as

$$
\begin{aligned}
P(z) & =P_{m}+\left(P_{c}-P_{m}\right) \frac{V_{c}}{1+V_{m}\left(\frac{P_{c}}{P_{m}}-1\right) \frac{1+v}{3-3 v}}, \\
V_{c} & =\left(\frac{1}{2}+\frac{z}{h}\right)^{k}, V_{m}=1-V_{c} .
\end{aligned}
$$

An exponential law is introduced by Delale and Erdogan [44] as follows

$$
P(z)=P_{m} \mathrm{e}^{p\left(z+\frac{h}{2}\right)}, \quad p=\frac{1}{h} \ln \left(\frac{P_{c}}{P_{m}}\right),
$$

where $p$ is the volume fraction exponent. In the present study, we restricted our attentions to the formula given in Eq. (1).

\subsection{Refined quasi-3D shear and normal deformation theory}

Shimpi and Patel [45] and Zenkour [46-49] introduced a refined shear deformation plate theory according to the following displacements field

$$
u_{1}=u-z \frac{\partial w^{b}}{\partial x}-f(z) \frac{\partial w^{s}}{\partial x},
$$




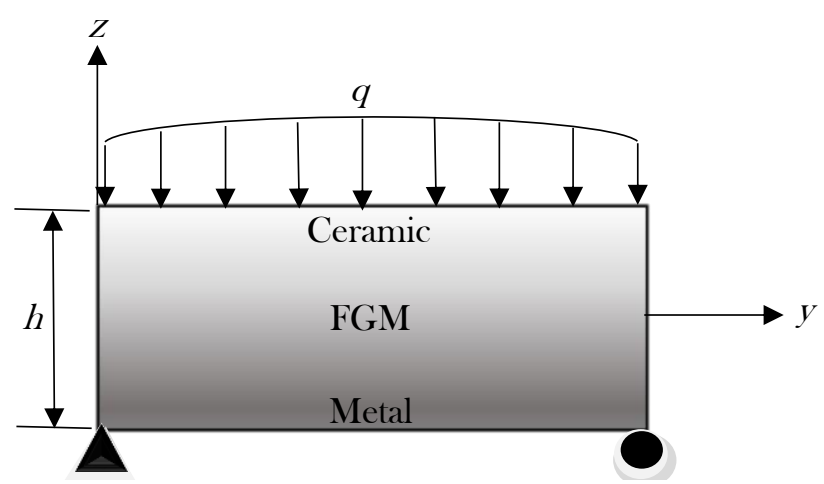

Figure 1: Geometry of the FG plate.

$$
\begin{aligned}
& u_{2}=v-z \frac{\partial w^{b}}{\partial y}-f(z) \frac{\partial w^{s}}{\partial y}, \\
& u_{3}=w^{b}+w^{s}
\end{aligned}
$$

Concerning the effect of transverse normal stain in $z$ direction, Thai and Kim [50] added one unknown $\phi_{z}$ into the displacements field in Eq. (4) as follows

$$
\begin{aligned}
& u_{1}=u-z \frac{\partial w^{b}}{\partial x}+f(z) \frac{\partial w^{s}}{\partial x} \\
& u_{2}=v-z \frac{\partial w^{b}}{\partial y}+f(z) \frac{\partial w^{s}}{\partial y} \\
& u_{3}=w^{b}+w^{s}+g(z) \phi_{z}
\end{aligned}
$$

In this study we suggest the displacements field as follows

$$
\begin{aligned}
& u_{1}=u-z \frac{\partial w^{b}}{\partial x}-f(z) \frac{\partial w^{s}}{\partial x} \\
& u_{2}=v-z \frac{\partial w^{b}}{\partial y}-f(z) \frac{\partial w^{s}}{\partial y} \\
& u_{3}=w^{b}+\left(1+\alpha g^{\prime}(z)\right) w^{s}
\end{aligned}
$$

where $u$ and $v$ are the in-plane displacements in the directions of $x$ and $y$, respectively; $w^{b}$ and $w^{s}$ are the transverse bending and shear displacement components, respectively. This proposed third-order shear deformation theory may be named it a quasi-3D shear and normal deformation theory since it presented the best distributions for the transverse shear and normal strains through the thickness of the plate $\left(\sigma_{z} \neq 0\right.$ corresponding to $\varepsilon_{z} \neq 0$ ) without the need to use shear correction factors. Actually, this what we get if we use a 3D elasticity theory. The values of the parameter $\alpha$ takes zero to obtain the shear deformation theory as in Eq. (4) and one for the suggested refined quasi-3D shear and normal deformation theory. The polynomial shape function is used as Thai and Kim [51]; $f(z)=-\frac{z}{4}+\frac{5}{3} \frac{z^{3}}{h^{2}}$. By numerical experiments, the function $g(z)=\frac{1}{12}(z-f(z))$ is given. The present refined quasi$3 \mathrm{D}$ shear and normal deformation model satisfies the free transverse shear stress condition on the upper and lower surfaces of the plate

$$
\tau_{y z}=\tau_{x z}=0 \quad z= \pm \frac{h}{2} .
$$

According to the displacement field in Eq. (6), the strain components can be expressed

$$
\begin{aligned}
\left\{\begin{array}{c}
\varepsilon_{x} \\
\varepsilon_{y} \\
\gamma_{x y}
\end{array}\right\} & =\left\{\begin{array}{c}
\varepsilon_{x}^{0} \\
\varepsilon_{y}^{0} \\
\gamma_{x y}^{0}
\end{array}\right\}+z\left\{\begin{array}{c}
\kappa_{x}^{b} \\
\kappa_{y}^{b} \\
\kappa_{x y}^{b}
\end{array}\right\}+f(z)\left\{\begin{array}{c}
\kappa_{x}^{s} \\
\kappa_{y}^{s} \\
\kappa_{x y}^{s}
\end{array}\right\}, \\
\left\{\begin{array}{c}
\gamma_{y z} \\
\gamma_{x z}
\end{array}\right\} & =\left[1-f^{\prime}(z)+\alpha g^{\prime}(z)\right]\left\{\begin{array}{c}
\gamma_{y z}^{0} \\
\gamma_{x z}^{0}
\end{array}\right\}, \\
\varepsilon_{z} & =\alpha g^{\prime \prime}(z) \varepsilon_{z}^{0} .
\end{aligned}
$$

where

$$
\begin{aligned}
& \varepsilon_{x}^{0}=\frac{\partial u}{\partial x}, \quad \varepsilon_{y}^{0}=\frac{\partial v}{\partial y}, \quad \gamma_{x y}^{0}=\frac{\partial u}{\partial y}+\frac{\partial v}{\partial x}, \\
& \varepsilon_{z}^{0}=w^{s}, \quad \gamma_{y z}^{0}=\frac{\partial w^{s}}{\partial y}, \\
& \gamma_{x z}^{0}=\frac{\partial w^{s}}{\partial x}, \quad \kappa_{x}^{b}=-\frac{\partial^{2} w^{b}}{\partial x^{2}}, \quad \kappa_{y}^{b}=-\frac{\partial^{2} w^{b}}{\partial y^{2}}, \\
& \kappa_{x y}^{b}=-2 \frac{\partial^{2} w^{b}}{\partial x \partial y}, \quad \kappa_{x}^{s}=-\frac{\partial^{2} w^{s}}{\partial x^{2}}, \\
& \kappa_{y}^{s}=-\frac{\partial^{2} w^{s}}{\partial y^{2}}, \quad \kappa_{x y}^{s}=-2 \frac{\partial^{2} w^{s}}{\partial x \partial y} .
\end{aligned}
$$

\subsection{Constitutive equations}

The linear constitutive relations for the FG plate can be expressed as

$$
\left\{\begin{array}{c}
\sigma_{x} \\
\sigma_{y} \\
\sigma_{z} \\
\tau_{y z} \\
\tau_{x z} \\
\tau_{x y}
\end{array}\right\}=\left[\begin{array}{cccccc}
c_{11} & c_{12} & c_{13} & 0 & 0 & 0 \\
c_{12} & c_{22} & c_{23} & 0 & 0 & 0 \\
c_{12} & c_{23} & c_{33} & 0 & 0 & 0 \\
0 & 0 & 0 & c_{44} & 0 & 0 \\
0 & 0 & 0 & 0 & c_{55} & 0 \\
0 & 0 & 0 & 0 & 0 & c_{66}
\end{array}\right]\left\{\begin{array}{c}
\varepsilon_{x} \\
\varepsilon_{y} \\
\varepsilon_{z} \\
\gamma_{y z} \\
\gamma_{x z} \\
\gamma_{x y}
\end{array}\right\},
$$

where the elastic constants $c_{i j}$ depend on the normal strain, therefore when $\alpha=1, c_{i j}$ are

$$
\begin{aligned}
& c_{11}=c_{22}=c_{33}=\frac{E\left(1-v^{2}\right)}{1-3 v^{2}-2 v^{3}}, \\
& c_{12}=c_{13}=c_{23}=\frac{v E(1+v)}{1-3 v^{2}-2 v^{3}}, \\
& c_{44}=c_{55}=c_{66}=\frac{E}{2(1+v)},
\end{aligned}
$$

whereas for $\alpha=0, c_{i j}$ are

$$
\begin{aligned}
& c_{11}=c_{22}=c_{33}=\frac{E}{\left(1-v^{2}\right)}, \\
& c_{12}=c_{13}=c_{23}=\frac{v E}{\left(1-v^{2}\right)},
\end{aligned}
$$




$$
c_{44}=c_{55}=c_{66}=\frac{E}{2(1+v)} \text {. }
$$

The stress resultants $N_{i j}$, the stress couples $M_{i j}$, the additional stress couples $S_{i j}$, the transverse shear stress resultants $Q_{i z}$ and the additional stress couples associated with transverse normal deformation effect $S_{z}$ can be gotten by integrating Eq. (10) over the plate thickness, and are expressed as

$$
\begin{aligned}
& \left\{\begin{array}{c}
N_{x} \\
N_{y} \\
M_{x} \\
M_{y} \\
S_{x} \\
S_{y} \\
S_{z}
\end{array}\right\}= \\
& {\left[\begin{array}{lllllll}
A_{11} & A_{12} & B_{11} & B_{12} & C_{11} & C_{12} & D_{13} \\
A_{12} & A_{22} & B_{12} & B_{22} & C_{12} & C_{22} & D_{23} \\
B_{11} & B_{12} & E_{11} & E_{12} & F_{11} & F_{12} & G_{13} \\
B_{12} & B_{22} & E_{12} & E_{22} & F_{12} & F_{22} & G_{23} \\
C_{11} & C_{12} & F_{11} & F_{12} & H_{11} & H_{12} & I_{13} \\
C_{12} & C_{22} & F_{12} & F_{22} & H_{12} & H_{22} & I_{23} \\
D_{13} & D_{23} & G_{13} & G_{23} & I_{13} & I_{23} & K_{33}
\end{array}\right] \times\left\{\begin{array}{c}
\varepsilon_{x}^{0} \\
\varepsilon_{y}^{0} \\
\kappa_{x}^{b} \\
\kappa_{y}^{b} \\
\kappa_{x}^{s} \\
\kappa_{y}^{s} \\
\varepsilon_{z}^{0}
\end{array}\right\},} \\
& \left\{\begin{array}{c}
N_{x y} \\
M_{x y} \\
S_{x y}
\end{array}\right\}=\left[\begin{array}{lll}
A_{66} & B_{66} & C_{66} \\
B_{66} & E_{66} & F_{66} \\
C_{66} & F_{66} & H_{66}
\end{array}\right]\left\{\begin{array}{l}
\gamma_{x y}^{0} \\
\kappa_{x y}^{b} \\
\kappa_{x y}^{b}
\end{array}\right\}, \\
& \left\{Q_{y z}, Q_{x z}\right\}=\left\{J_{44} \gamma_{y z}^{0}, J_{55} \gamma_{x z}^{0}\right\},
\end{aligned}
$$

and the coefficients $A_{i j}, B_{i j}, C_{i j}, \cdots$, etc., are defined by

$$
\begin{aligned}
&\left\{A_{i j}, B_{i j}, E_{i j}, C_{i j}\right\}=\int_{-\frac{h}{2}}^{\frac{h}{2}} c_{i j}\left\{1, z, z^{2}, f(z)\right\} \mathrm{d} z, \\
& i, j= 1,2,6, \\
&\left\{D_{i 3}, G_{i 3}, I_{i 3}\right\}= \int_{-\frac{h}{2}}^{\frac{h}{2}} \alpha c_{i j} g^{\prime \prime}(z)\{1, z, f(z)\} \mathrm{d} z, i=1,2, \\
&\left\{F_{i j}, H_{i j}\right\}= \int_{-\frac{h}{2}}^{\frac{h}{2}} c_{i j} f(z)\{z, f(z)\} \mathrm{d} z, i, j=1,2,6, \\
&\left\{J_{44}, J_{55}\right\}= \int_{-\frac{h}{2}}^{\frac{h}{2}}\left\{c_{44}, c_{55}\right\}\left[1-f^{\prime}(z)+\alpha g^{\prime}(z)\right]^{2} \mathrm{~d} z, \\
& K_{33}= \int_{-\frac{h}{2}}^{\frac{h}{2}} c_{33}\left[\alpha g^{\prime \prime}(z)\right]^{2} \mathrm{~d} z .
\end{aligned}
$$

\subsection{Governing equations}

According to the principle of virtual work the governing equations of equilibrium can be derived by

$$
\iiint_{V} \sigma_{i j} \delta \varepsilon_{i j} \mathrm{~d} v-\iint_{\Omega} q\left(\delta w^{b}+\delta w^{s}\right) \mathrm{d} \Omega=0 .
$$

By using the strain and displacement components in Eqs. (6), (8) and (9) and integrate over the plate thickness and then collecting terms, Eq. (15) can be written as

$$
\begin{aligned}
& \iint_{\Omega}\left[N_{x} \frac{\partial \delta u}{\partial x}-M_{x} \frac{\partial^{2} \delta w^{b}}{\partial x^{2}}-S_{x} \frac{\partial^{2} \delta w^{s}}{\partial x^{2}}\right. \\
& +N_{y} \frac{\partial \delta v}{\partial y}-M_{y} \frac{\partial^{2} \delta w^{b}}{\partial y^{2}}-S_{y} \frac{\partial^{2} \delta w^{s}}{\partial y^{2}}+S_{z} \delta w^{s} \\
& +Q_{y z} \frac{\partial \delta w^{s}}{\partial y}+Q_{x z} \frac{\partial \delta w^{s}}{\partial x}+N_{x y}\left(\frac{\partial \delta u}{\partial y}+\frac{\partial \delta v}{\partial x}\right) \\
& \left.-2 M_{x y} \frac{\partial^{2} \delta w^{b}}{\partial x \partial y}-2 S_{x y} \frac{\partial^{2} \delta w^{s}}{\partial x \partial y}-q \delta w^{b}-q \delta w^{s}\right] \mathrm{d} \Omega=0
\end{aligned}
$$

The equilibrium equations are obtained from Eq. (16) by integrating by parts and then equating the coefficients of $\delta u, \delta v, \delta w^{b}$ and $\delta w^{s}$ to zero, individually. The equilibrium equations related with the present theory are

$$
\begin{aligned}
& \delta u: \frac{\partial N_{x}}{\partial x}+\frac{\partial N_{x y}}{\partial y}=0 \\
& \delta v: \frac{\partial N_{x y}}{\partial x}+\frac{\partial N_{y}}{\partial y}=0 \\
& \delta w^{b}: \quad \frac{\partial^{2} M_{x}}{\partial x^{2}}+2 \frac{\partial^{2} M_{x y}}{\partial x \partial y}+\frac{\partial^{2} M_{y}}{\partial y^{2}}+q=0, \\
& \delta w^{s}: \quad \frac{\partial^{2} S_{x}}{\partial x^{2}}+2 \frac{\partial^{2} S_{x y}}{\partial x \partial y}+\frac{\partial^{2} S_{y}}{\partial y^{2}}+\frac{\partial Q_{x z}}{\partial x}+ \\
& \frac{\partial Q_{y z}}{\partial y}-S_{z}+q=0 .
\end{aligned}
$$

Expressing the stress resultants in terms of displacements, the equilibrium equations become

$$
\begin{aligned}
& A_{11} \frac{\partial^{2} u}{\partial x^{2}}-B_{11} \frac{\partial^{3} w^{b}}{\partial x^{3}}-C_{11} \frac{\partial^{3} w^{s}}{\partial x^{3}}+A_{12} \frac{\partial^{2} v}{\partial x \partial y} \\
& -B_{12} \frac{\partial^{3} w^{b}}{\partial x \partial y^{2}}-C_{12} \frac{\partial^{3} w^{s}}{\partial x \partial y^{2}}+D_{13} \frac{\partial w^{s}}{\partial x}+ \\
& A_{66}\left(\frac{\partial^{2} u}{\partial y^{2}}+\frac{\partial^{2} v}{\partial x \partial y}\right)-2 B_{66} \frac{\partial^{3} w^{b}}{\partial x \partial y^{2}}-2 C_{66} \frac{\partial^{3} w^{s}}{\partial x \partial y^{2}}=0, \\
& A_{66}\left(\frac{\partial^{2} u}{\partial x \partial y}+\frac{\partial^{2} v}{\partial x^{2}}\right)-2 B_{66} \frac{\partial^{3} w^{b}}{\partial x \partial y^{2}}-2 C_{66} \frac{\partial^{3} w^{s}}{\partial x \partial y^{2}} \\
& +A_{12} \frac{\partial^{2} u}{\partial x \partial y}-B_{12} \frac{\partial^{3} w^{b}}{\partial x \partial y^{2}}-C_{12} \frac{\partial^{3} w^{S}}{\partial x \partial y^{2}}+A_{22} \frac{\partial^{2} v}{\partial y^{2}}- \\
& B_{22} \frac{\partial^{3} w^{b}}{\partial y^{3}}-C_{22} \frac{\partial^{3} w^{s}}{\partial y^{3}}+D_{23} \frac{\partial w^{s}}{\partial y}=0,
\end{aligned}
$$




$$
\begin{aligned}
& B_{11} \frac{\partial^{3} u}{\partial x^{3}}-E_{11} \frac{\partial^{4} w^{b}}{\partial x^{4}}-F_{11} \frac{\partial^{4} w^{s}}{\partial x^{4}}+B_{12} \frac{\partial^{3} v}{\partial x \partial y^{2}} \\
& -2 E_{12} \frac{\partial^{4} w^{b}}{\partial x^{2} \partial y^{2}}-2 F_{12} \frac{\partial^{4} w^{s}}{\partial x^{2} \partial y^{2}}+G_{13} \frac{\partial^{2} w^{s}}{\partial x^{2}} \\
& +2 B_{66}\left(\frac{\partial^{3} u}{\partial x \partial y^{2}}+\frac{\partial^{3} v}{\partial x^{2} \partial y}\right)-4 E_{66}\left(\frac{\partial^{4} w^{b}}{\partial x^{2} \partial y^{2}}+\frac{\partial^{4} w^{s}}{\partial x^{2} \partial y^{2}}\right) \\
& +B_{12} \frac{\partial^{3} u}{\partial x \partial y^{2}}+B_{22} \frac{\partial^{3} v}{\partial x^{3}}-E_{22} \frac{\partial^{4} w^{b}}{\partial x^{4}}+G_{23} \frac{\partial^{2} w^{s}}{\partial y^{2}}+q=0, \\
& C_{66} \frac{\partial^{3} u}{\partial x \partial y^{2}}+C_{22} \frac{\partial^{3} v}{\partial y^{3}}-F_{22} \frac{\partial^{4} w^{b}}{\partial y^{4}}-H_{22} \frac{\partial^{4} w^{s}}{\partial y^{4}}+ \\
& J_{55} \frac{\partial^{2} w^{s}}{\partial x^{2}}+C_{11} \frac{\partial^{3} u}{\partial x^{3}}-F_{11} \frac{\partial^{4} w^{b}}{\partial x^{4}}-H_{11} \frac{\partial^{4} w^{s}}{\partial x^{4}} \\
& +C_{12} \frac{\partial^{3} v}{\partial x^{2} \partial y}-2 F_{22} \frac{\partial^{4} w^{b}}{\partial x^{2} \partial y^{2}}-2 H_{12} \frac{\partial^{4} w^{s}}{\partial x^{2} \partial y^{2}} \\
& +2 C_{66}\left(\frac{\partial^{3} u}{\partial x \partial y^{2}}+\frac{\partial^{3} v}{\partial x^{2} \partial y}\right)+C_{12} \frac{\partial^{3} v}{\partial x \partial y^{2}}- \\
& 4 F_{66} \frac{\partial^{4} w^{b}}{\partial x^{2} \partial y^{2}}-4 H_{66} \frac{\partial^{4} w^{s}}{\partial x^{2} \partial y^{2}}-D_{23} \frac{\partial v}{\partial y}-K_{33} w^{s}+G_{13} \frac{\partial^{2} w^{b}}{\partial x^{2}} \\
& +2 I_{13} \frac{\partial^{2} w^{s}}{\partial x^{2}}+G_{23} \frac{\partial^{2} w^{b}}{\partial y^{2}}+2 I_{23} \frac{\partial^{2} w^{s}}{\partial y^{2}}-D_{13} \frac{\partial u}{\partial y}+q=0 .
\end{aligned}
$$

\subsection{Closed-form solution}

Rectangular plates mostly categorized for the support used kind. Here we search for exact solutions of Eq. (17) with simply-supported FG plate. The following boundary conditions are considered at the FG plate side edges:

$$
\begin{gathered}
v=w^{b}=w^{s}=\frac{\partial w^{b}}{\partial y}=\frac{\partial w^{s}}{\partial y}= \\
N_{x}=M_{x}=S_{x}=S_{z}=0, \text { at } x=0, a, \\
u=w^{b}=w^{s}=\frac{\partial w^{b}}{\partial x}=\frac{\partial w^{s}}{\partial x}= \\
N_{y}=M_{y}=S_{y}=S_{z}=0, \text { at } y=0, b .
\end{gathered}
$$

The mechanical load $q$, the bending component $w^{b}$ and the shear component $w^{s}$ of the transverse displacements $u_{3}$ follows Navier's solution as follows

$$
\left\{\begin{array}{c}
q \\
w^{b} \\
w^{s}
\end{array}\right\}=\left\{\begin{array}{c}
q_{0} \\
W^{b} \\
W^{s}
\end{array}\right\} \sin (\lambda x) \sin (\mu y),
$$

where $\lambda=\frac{\pi}{a}, \mu=\frac{\pi}{b}$ and $q_{0}$ is the intensity of the load at the center of the plate. The in-plane displacements $u$ and $v$ are supposed to be of the following form

$$
\left\{\begin{array}{l}
u \\
v
\end{array}\right\}=\left\{\begin{array}{l}
U \cos (\lambda x) \sin (\mu y) \\
V \sin (\lambda x) \cos (\mu y)
\end{array}\right\}
$$

in which $U, V, W^{b}$ and $W^{s}$ are arbitrary parameters will be determined. Using Eq. (20) and Eq. (21) in Eqs. (18) we get

$$
[A]\{\Delta\}=\{F\},
$$

where $\{\Delta\}$ and $\{F\}$ are the following columns

$$
\{\Delta\}=\left\{U, V, W^{b}, W^{s}\right\}, \quad\{F\}=\left\{0,0, q_{0}, q_{0}\right\},
$$

and the symmetric elements of matrix $[A]$ are expressed as

$$
\begin{aligned}
a_{11} & =A_{11} \lambda^{2}+A_{66} \mu^{2}, \\
a_{12} & =\left(A_{12}+A_{66}\right) \lambda \mu, \\
a_{13} & =-\lambda\left[B_{11} \lambda^{2}+\left(B_{12}+2 B_{66}\right) \mu^{2}\right], \\
a_{14} & =-\lambda\left[C_{11} \lambda^{2}+\left(C_{12}+2 C_{66}\right) \mu^{2}+D_{13}\right], \\
a_{22} & =A_{66} \lambda^{2}+A_{22} \mu^{2}, \\
a_{23} & =-\mu\left[\left(B_{12}+2 B_{66}\right) \lambda^{2}+B_{22} \mu\right], \\
a_{24} & =-\mu\left[C_{22} \mu^{2}+\left(C_{12}+2 C_{66}\right) \lambda^{2}+D_{23}\right], \\
a_{33} & =E_{11} \lambda^{4}+2\left(E_{12}+2 E_{66}\right) \lambda^{2} \mu^{2}+E_{22} \mu^{4}, \\
a_{34} & =F_{11} \lambda^{4}+2\left(F_{12}+2 F_{66}\right) \lambda^{2} \mu^{2}+F_{22} \mu^{4} \\
& +G_{13} \lambda^{2}+G_{23} \mu^{2}, \\
a_{44} & =H_{11} \lambda^{4}+2\left(H_{12}+2 F_{66}\right) \lambda^{2} \mu^{2}+H_{22} \mu^{4} \\
& +2 I_{13} \lambda^{2}+2 I_{23} \mu^{2}+J_{55} \lambda^{2}+J_{44} \mu^{2}+K_{33} .
\end{aligned}
$$

\section{Numerical results and discussions}

A refined quasi-3D shear and normal deformation theory is used to analyze the bending of a simply-supported rectangular plate under sinusoidally distributed transverse mechanical load applied at the upper surface of the plate. The FG plate is graded from the lower surface (metal) to the upper surface (ceramic) and the mixture rule is used based on Eq. (1). Young's modulus for metal (Aluminium, $\mathrm{Al}$ ) is $70 \times 10^{9} \mathrm{~N} / \mathrm{m}^{2}$ while for ceramic (Alumina, $\mathrm{Al}_{2} \mathrm{O}_{3}$ ) is $380 \times 10^{9} \mathrm{~N} / \mathrm{m}^{2}$. The Poisson's ratio is fixed at 0.3 . The applied non-dimensional parameters are

$$
\begin{aligned}
& \bar{u}_{1}=\frac{100 h^{3} E_{c}}{a^{4} q_{0}} u_{1}\left(\frac{a}{2}, \frac{b}{2}, \bar{z}\right), \\
& \bar{u}_{2}=\frac{100 h^{3} E_{c}}{a^{4} q_{0}} u_{2}\left(\frac{a}{2}, \frac{b}{2}, \bar{z}\right), \\
& \bar{u}_{3}=\frac{10 h^{3} E_{c}}{a^{4} q_{0}} u_{3}\left(\frac{a}{2}, \frac{b}{2}, \bar{z}\right), \\
& \bar{\sigma}_{x}=\frac{h}{a q_{0}} \sigma_{x}\left(\frac{a}{2}, \frac{b}{2}, \bar{z}\right), \quad \bar{\sigma}_{y}=\frac{h}{a q_{0}} \sigma_{y}\left(\frac{a}{2}, \frac{b}{2}, \bar{z}\right), \\
& \bar{\sigma}_{z}=\frac{1}{q_{0}} \sigma_{z}\left(\frac{a}{2}, \frac{b}{2}, \bar{z}\right), \quad \bar{\tau}_{y z}=\frac{h}{a q_{0}} \tau_{y z}\left(\frac{a}{2}, 0, \bar{z}\right), \\
& \bar{\tau}_{x z}=\frac{h}{a q_{0}} \tau_{x z}\left(0, \frac{b}{2}, \bar{z}\right), \\
& \bar{\tau}_{x y}=\frac{h}{a q_{0}} \tau_{x y}(0,0, \bar{z}), \quad \bar{z}=\frac{z}{h} .
\end{aligned}
$$


Table 1: Effect of $\varepsilon_{z}$ on the non-dimensional displacement $\bar{u}_{3}$ and axial stress $\bar{\sigma}_{x}$ of a FG square plate.

\begin{tabular}{|c|c|c|c|c|c|c|c|c|}
\hline \multirow[t]{2}{*}{$k$} & \multirow[t]{2}{*}{ Source } & \multirow[t]{2}{*}{$\varepsilon_{z}$} & \multicolumn{3}{|c|}{$\bar{u}_{3}(0)$} & \multicolumn{3}{|c|}{$\bar{\sigma}_{x}(1 / 3)$} \\
\hline & & & $a / h=4$ & $a / h=10$ & $a / h=100$ & $a / h=4$ & $a / h=10$ & $a / h=100$ \\
\hline \multirow[t]{7}{*}{1} & CPT [21] & $=0$ & 0.5623 & 0.5623 & 0.5623 & 0.8060 & 2.0150 & 20.150 \\
\hline & FPT [21] & $=0$ & 0.7291 & 0.5889 & 0.5625 & 0.8060 & 2.0150 & 20.150 \\
\hline & Present & $=0$ & 0.7984 & 0.5889 & 0.5625 & 0.5819 & 1.4898 & 14.968 \\
\hline & Carrera et al. [22] & $\neq 0$ & 0.7171 & 0.5845 & 0.5624 & 0.6221 & 1.5064 & 14.969 \\
\hline & Neves et al. [23] & $\neq 0$ & 0.6997 & 0.5845 & 0.5624 & 0.5925 & 1.4962 & 14.969 \\
\hline & Zenkour [24] & $\neq 0$ & 0.6828 & 0.5592 & 0.5624 & 0.5944 & 1.4962 & 14.552 \\
\hline & Present & $\neq 0$ & 0.6828 & 0.5592 & 0.5459 & 0.5945 & 1.4966 & 14.558 \\
\hline \multirow[t]{7}{*}{4} & CPT [21] & $=0$ & 0.8281 & 0.8281 & 0.8281 & 0.6420 & 1.6049 & 16.049 \\
\hline & FPT [21] & $=0$ & 1.1125 & 0.8736 & 0.8281 & 0.6420 & 1.6049 & 16.049 \\
\hline & Present & $\neq 0$ & 1.1598 & 0.8815 & 0.8287 & 0.4449 & 1.1794 & 11.921 \\
\hline & Carrera et al. [22] & $\neq 0$ & 1.1585 & 0.8821 & 0.8286 & 0.4877 & 1.1971 & 11.923 \\
\hline & Neves et al. [23] & $\neq 0$ & 1.1178 & 0.8750 & 0.8286 & 0.4404 & 1.1783 & 11.932 \\
\hline & Zenkour [24] & $\neq 0$ & 1.1001 & 0.8404 & 0.7933 & 0.4321 & 1.1410 & 11.388 \\
\hline & Present & $\neq 0$ & 1.1001 & 0.8404 & 0.7933 & 0.4324 & 1.1416 & 11.395 \\
\hline \multirow[t]{7}{*}{10} & CPT [21] & $=0$ & 0.9354 & 0.9354 & 0.9354 & 0.4796 & 1.1990 & 11.990 \\
\hline & FPT [21] & $=0$ & 1.3178 & 0.9966 & 0.9360 & 0.4796 & 1.1990 & 11.990 \\
\hline & Present & $=0$ & 1.3909 & 1.0087 & 0.9362 & 0.3259 & 0.8785 & 8.9059 \\
\hline & Carrera et al. [22] & $\neq 0$ & 1.3745 & 1.0072 & 0.9361 & 0.3695 & 0.8965 & 8.9077 \\
\hline & Neves et al. [23] & $\neq 0$ & 1.3490 & 0.8750 & 0.9361 & 0.3227 & 1.1783 & 11.932 \\
\hline & Zenkour [24] & $\neq 0$ & 1.3391 & 0.9806 & 0.9140 & 0.3154 & 0.8530 & 8.5853 \\
\hline & Present & $\neq 0$ & 1.3391 & 0.9806 & 0.9139 & 0.3156 & 0.8535 & 8.5914 \\
\hline
\end{tabular}
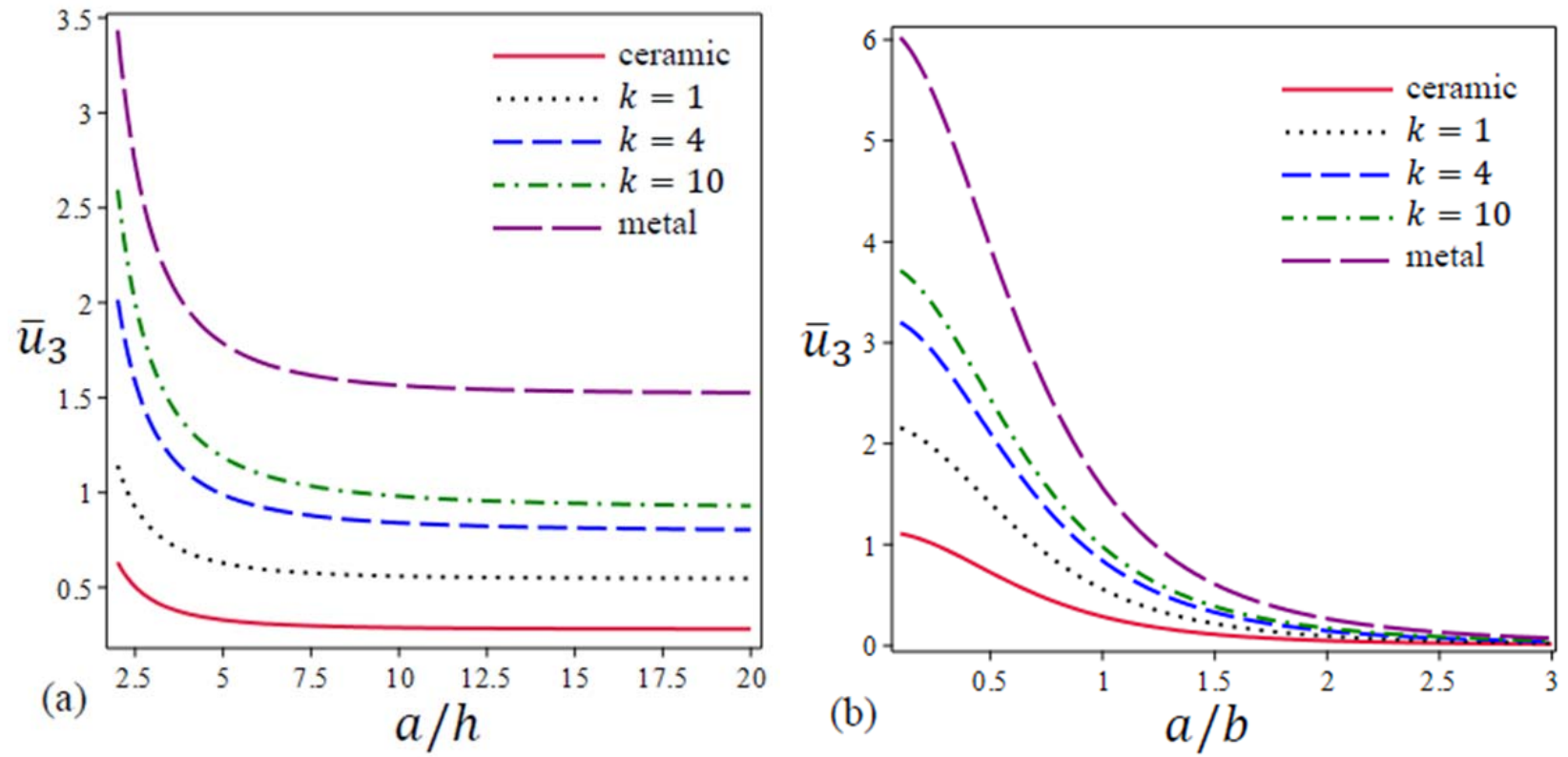

Figure 2: Non-dimensional displacement $\bar{u}_{3}$ as: (a) function of $a / h$, (b) function of $a / b$. 
Table 2: The non-dimensional displacements and stresses of a FG square plate.

\begin{tabular}{|c|c|c|c|c|c|c|c|c|c|c|}
\hline$k$ & Source & $\varepsilon_{z}$ & $\bar{u}_{1}\left(-\frac{1}{4}\right)$ & $\bar{u}_{2}\left(-\frac{1}{6}\right)$ & $\bar{u}_{3}(0)$ & $\bar{\sigma}_{X}\left(\frac{1}{2}\right)$ & $\bar{\sigma}_{y}\left(\frac{1}{3}\right)$ & $\overline{\boldsymbol{\tau}}_{y z}\left(\frac{1}{6}\right)$ & $\bar{\tau}_{x z}(0)$ & $\bar{\tau}_{x y}\left(-\frac{1}{3}\right)$ \\
\hline \multirow[t]{3}{*}{ Ceramic } & Zenkour [25] & $=0$ & 0.2309 & 0.1539 & 0.2960 & 1.9955 & 1.3121 & 0.2132 & 0.2462 & 0.7065 \\
\hline & Present & $=0$ & 0.2183 & 0.1451 & 0.2961 & 1.9943 & 1.3124 & 0.2121 & 0.2386 & 0.7067 \\
\hline & & $\neq 0$ & 0.2063 & 0.1369 & 0.2881 & 2.0635 & 1.3458 & 0.2963 & 0.3333 & 0.7067 \\
\hline \multirow[t]{3}{*}{1} & Zenkour [25] & $=0$ & 0.6626 & 0.5093 & 0.5889 & 3.0870 & 1.4894 & 0.2622 & 0.2642 & 0.6110 \\
\hline & Present & $=0$ & 0.6414 & 0.4944 & 0.5889 & 3.0850 & 1.4898 & 0.2608 & 0.2386 & 0.6111 \\
\hline & & $\neq 0$ & 0.5710 & 0.4348 & 0.5592 & 3.1738 & 1.4966 & 0.3643 & 0.3333 & 0.5486 \\
\hline \multirow[t]{3}{*}{2} & Zenkour [25] & $=0$ & 0.9281 & 0.7311 & 0.7573 & 3.6094 & 1.3954 & 0.2763 & 0.2265 & 0.5441 \\
\hline & Present & $=0$ & 0.8984 & 0.7102 & 0.7573 & 3.6067 & 1.3960 & 0.2737 & 0.2186 & 0.5442 \\
\hline & & $\neq 0$ & 0.7941 & 0.6198 & 0.7158 & 3.6812 & 1.3781 & 0.3501 & 0.2796 & 0.4853 \\
\hline \multirow[t]{3}{*}{4} & Zenkour [25] & $=0$ & 1.0941 & 0.8651 & 0.8819 & 4.0693 & 1.1783 & 0.2580 & 0.2029 & 0.5667 \\
\hline & Present & $=0$ & 1.0502 & 0.8342 & 0.8815 & 4.0655 & 1.1794 & 0.2537 & 0.1944 & 0.5669 \\
\hline & & $\neq 0$ & 0.9365 & 0.7334 & 0.8404 & 4.0935 & 1.1416 & 0.2858 & 0.2190 & 0.5104 \\
\hline \multirow[t]{3}{*}{10} & Zenkour [25] & $=0$ & 1.1372 & 0.8756 & 1.0089 & 5.0890 & 0.8775 & 0.2041 & 0.2198 & 0.5894 \\
\hline & Present & $=0$ & 1.0766 & 0.8329 & 1.0087 & 5.0849 & 0.8785 & 0.2014 & 0.2114 & 0.5896 \\
\hline & & $\neq 0$ & 0.9841 & 0.7498 & 0.9806 & 5.0870 & 0.8535 & 0.2172 & 0.2279 & 0.5443 \\
\hline \multirow[t]{3}{*}{ Metal } & Zenkour [25] & $=0$ & 1.2534 & 0.8356 & 1.6070 & 1.9955 & 1.3121 & 0.2132 & 0.2462 & 0.7065 \\
\hline & Present & $=0$ & 1.1851 & 0.7875 & 1.6072 & 1.9943 & 1.3124 & 0.2121 & 0.2386 & 0.7067 \\
\hline & & $\neq 0$ & 1.1199 & 0.7433 & 1.5642 & 2.0635 & 1.3458 & 0.2963 & 0.3333 & 0.6689 \\
\hline
\end{tabular}

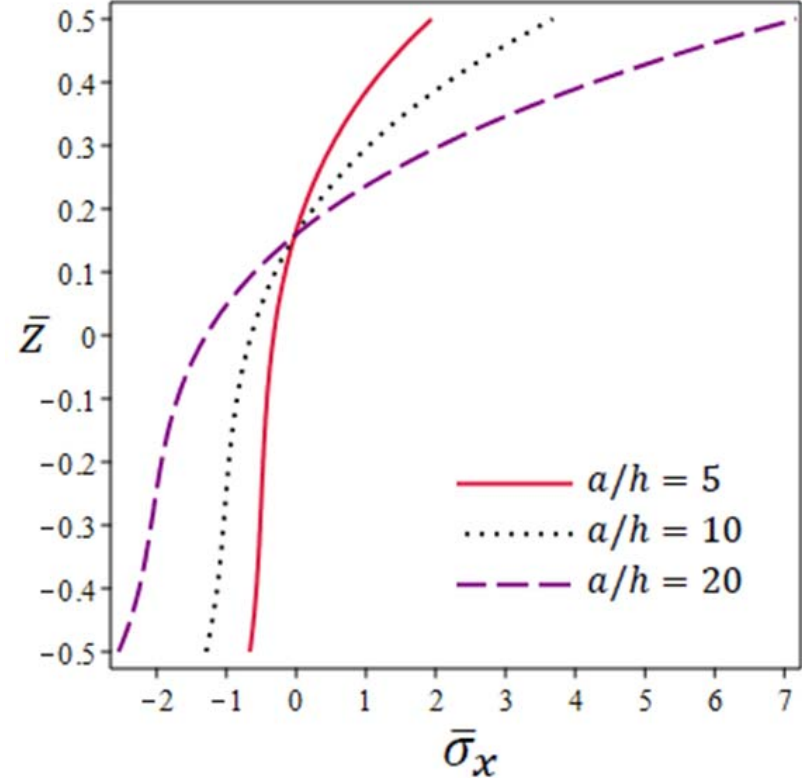

Figure 3: The axial stress $\bar{\sigma}_{x}$ across the thickness of a square FG plate with $k=2$.

We will suppose (except if we mentioned) that $a / h=5$, $a / b=2$. Table 1 shows numerical results for the nondimensional axial stress $\bar{\sigma}_{1}$ and the non-dimensional displacement $\bar{u}_{3}$ of a square plate. We considered three FG plates by using thick $(a / h=4)$, moderately thick $(a / h=$ $10)$ and thin $(a / h=100)$ plates with different volume

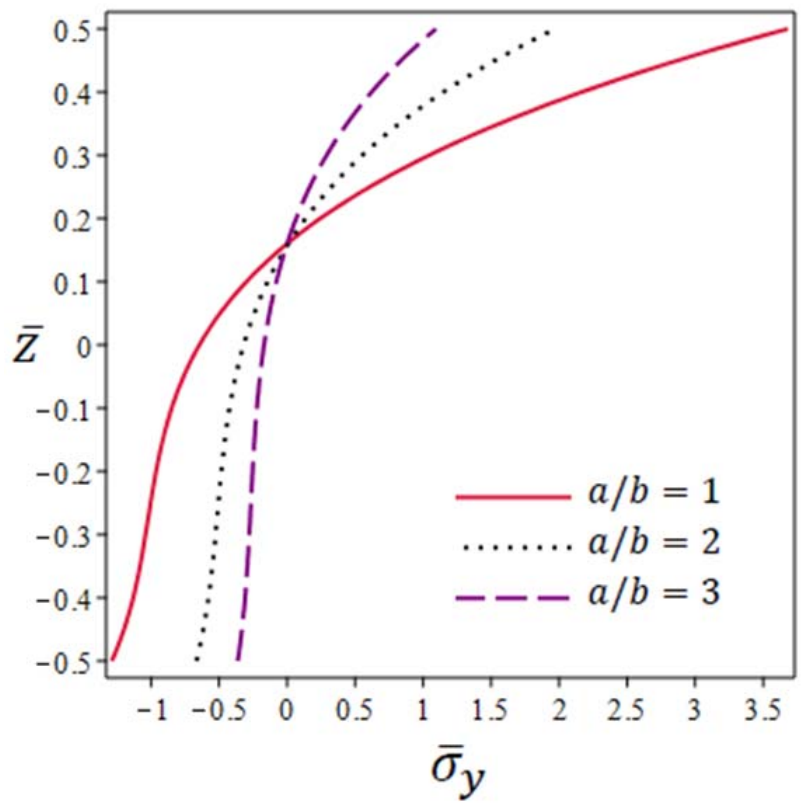

Figure 4: The axial stress $\bar{\sigma}_{y}$ across the thickness of a FG plate with $k=2$ and $a / h=10$.

fraction exponent. The present results of the shear deformation theory were compared with CPT and FPT of Zenkour [21] while the results of the refined quasi-3D shear and normal deformation theory were compared with the shear and normal deformation theories of Carrera et al. [22], Neves et al. [23] and Zenkour [24]. It can be seen that 


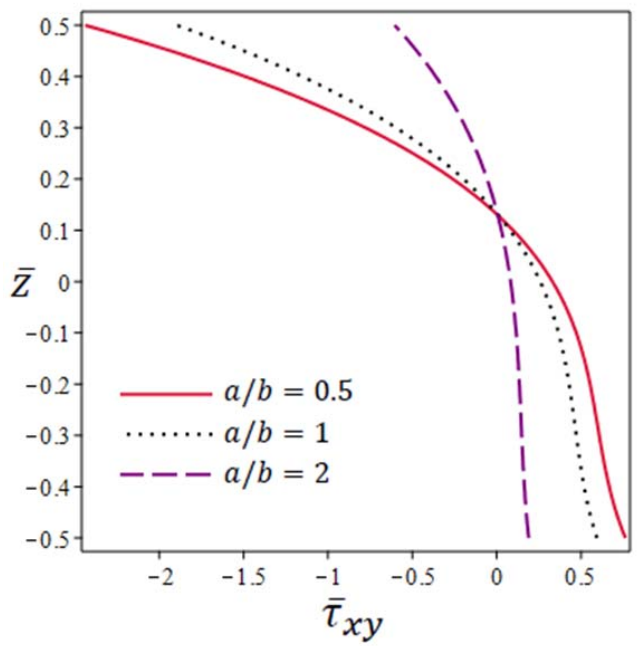

Figure 5: The tangential stress $\bar{\tau}_{x y}$ across the thickness of a FG plate with $k=2$ and $a / h=10$.
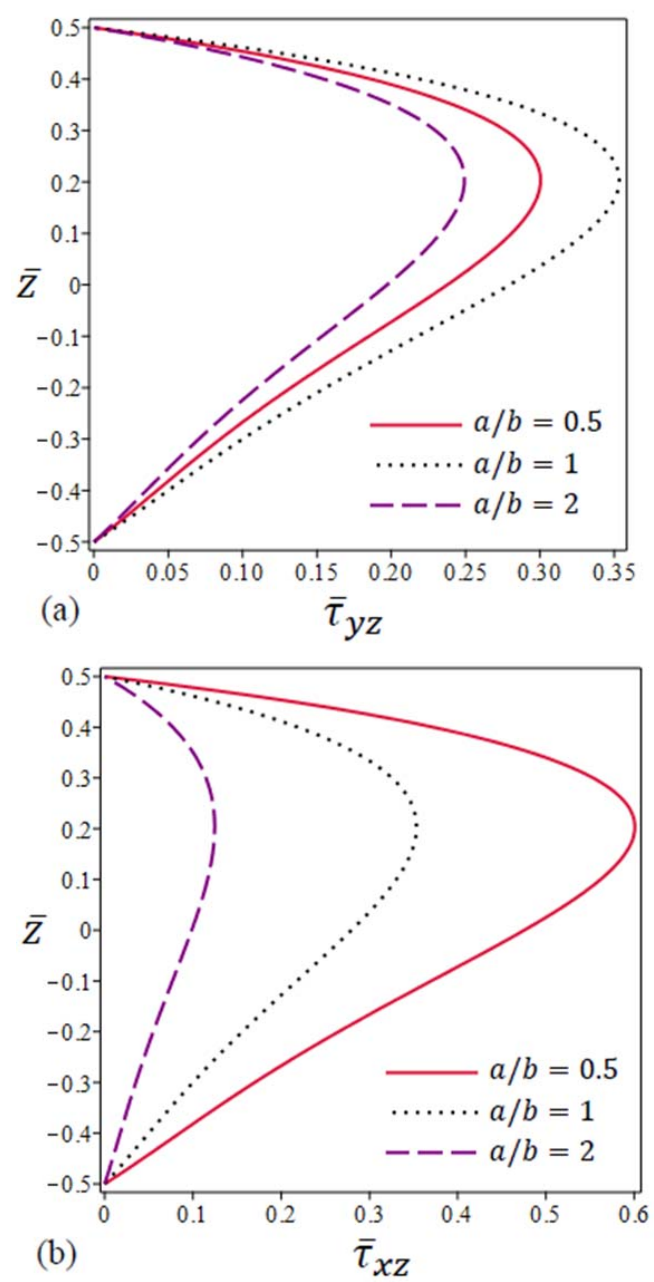

Figure 6: The transverse shear stresses across the thickness of a FG plate with $k=2$ and $a / h=10:$ (a) $\bar{\tau}_{y z}$, (b) $\bar{\tau}_{x z}$.
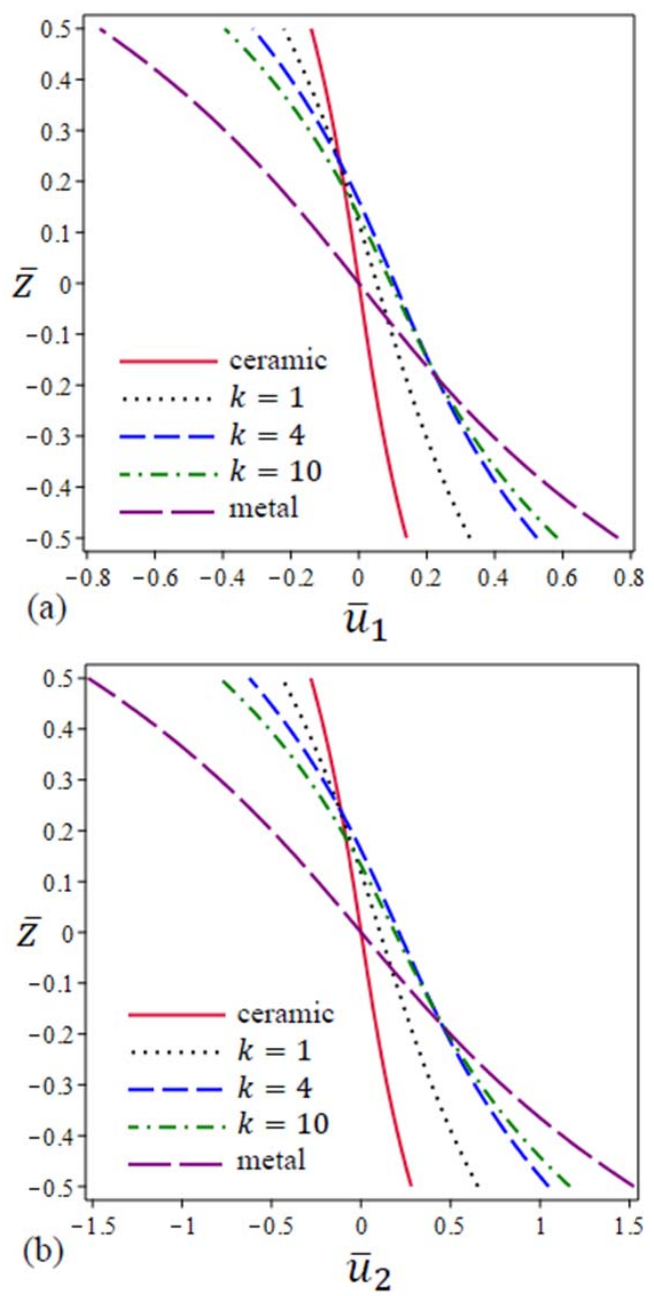

Figure 7: The in-plane displacements across the thickness of a FG plate: (a) $\bar{u}_{1}$, (b) $\bar{u}_{2}$.

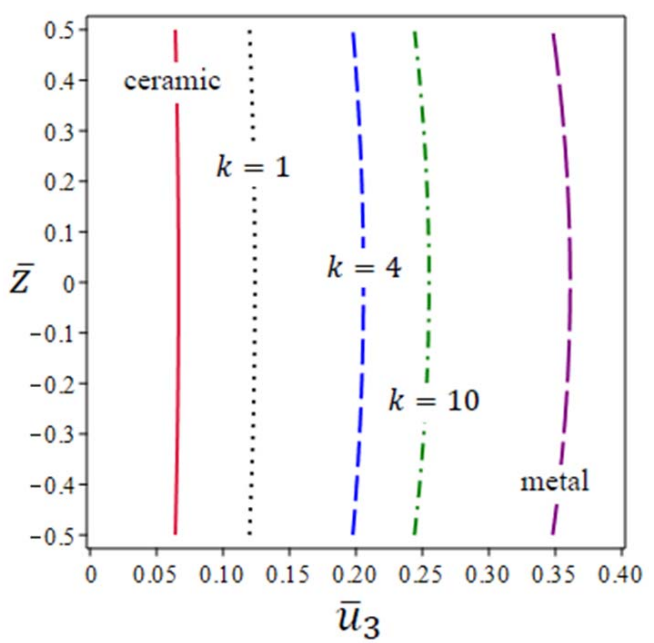

Figure 8: The transverse displacement across the thickness of a FG plate. 

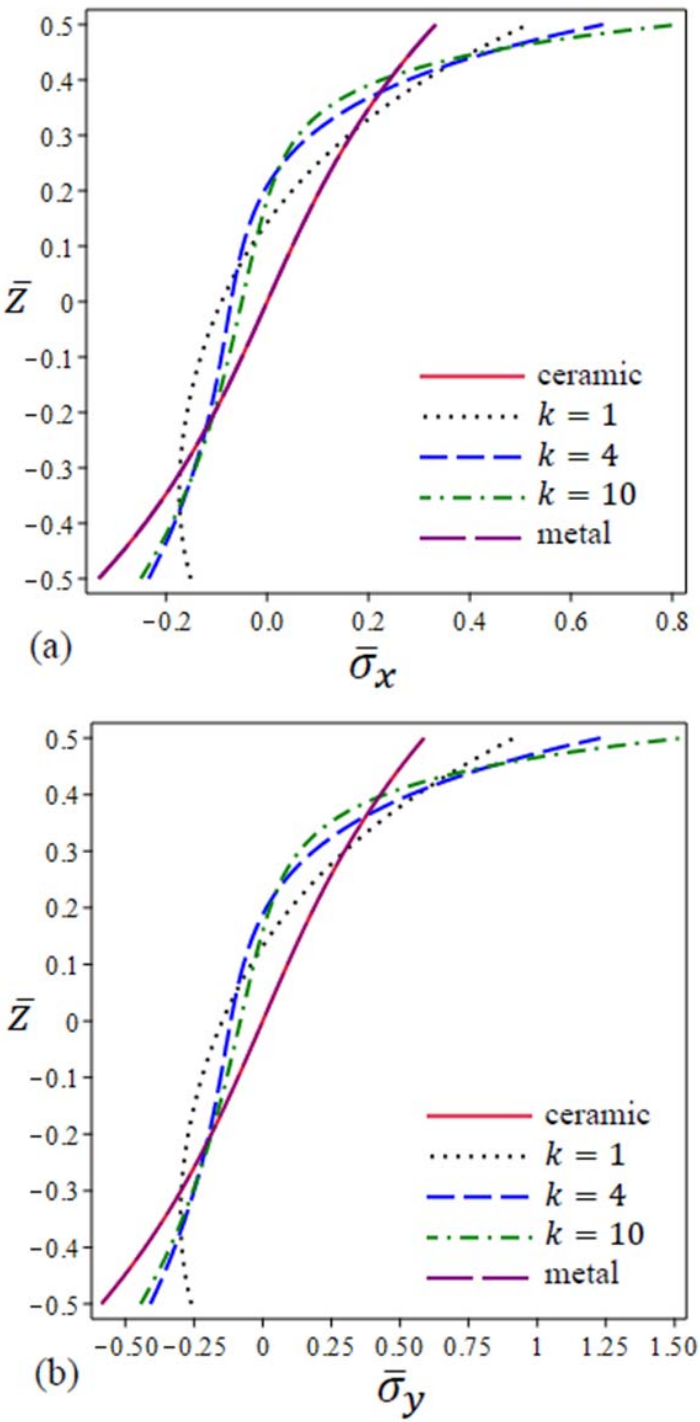

Figure 9: The in-plane axial stresses across the thickness of a FG plate: (a) $\bar{\sigma}_{x}$, (b) $\bar{\sigma}_{y}$.

the present results of the refined quasi-3D shear and normal deformation theory agree with the given theories. The non-dimensional displacement and stresses of a FG square plate $(a / h=10)$ are presented for different values of $k$ in Table 2. The present results were compared with the results given by Zenkour [25]. The present shear deformation theory agrees with those given by Zenkour [25] while the present refined quasi-3D shear and normal deformation theory gives more accurate results due to the effect of the normal strain $\varepsilon_{z}$ which produces a considerable change. Figure 2 shows the transverse center deflection variation with side-to-thickness and aspect ratios, respectively. Note that as the volume fraction exponent decreases the deflections also decreases. Figures 3 and 4 show the in-plane axial stresses across plate thickness. The stresses compress

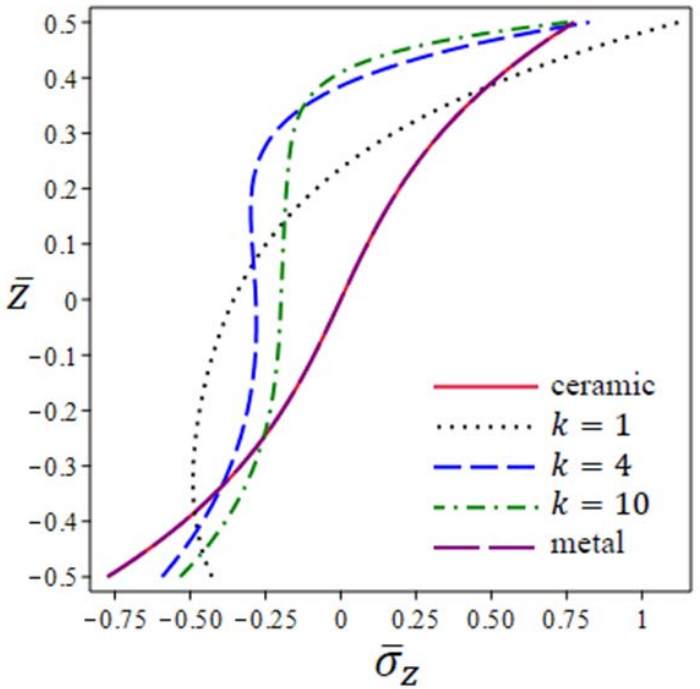

Figure 10: The transverse normal stress across the thickness of a FG plate.

across the plate up to $z \approx 0.164$ and then turn out to be tensile while the value for the tangential stress $\bar{\tau}_{x y}$ is $z \approx 0.142$ as shown in Figure 5. The distribution of the transverse shear stresses $\bar{\tau}_{y z}$ and $\bar{\tau}_{x y}$ across thickness is shown in Figure 6. It is obvious that the maximum value happens at $z \approx 0.21$ not at the plate center like the homogeneous plate. The distributions of displacements and stresses across the thickness of a FG rectangular plate under sinusoidal load for different volume fraction exponent values are presented in Figures 7-12. It can be observed that all the stresses of the fully ceramic and metal plates (homogeneous) are matched with each other because they are not depending on Young's modulus. The distributions of the in-plane displacements $\bar{u}_{1}$ and $\bar{u}_{2}$ across thickness gives a similar behavior as shown in Figure 7. Figure 8 displays the distributions of the transverse displacement $\bar{u}_{3}$ across thickness and it can be noted that the deflections of metal plate is more than the ceramic one. The distributions of the in-plane axial stresses $\bar{\sigma}_{x}$ and $\bar{\sigma}_{y}$ across the thickness of a FG plate exhibit similar behavior as shown in Figure 9 and it observed that these stresses are tensile at the upper surface while becoming compressive at the lower surface of the FG rectangular plate and the homogeneous plate gives the maximum compressive stresses at the lower surface and the minimum tensile stresses at the upper surface of the FG rectangular plate. It can be observed from Figure 10 that we cannot ignore the transverse normal stress $\bar{\sigma}_{z}$ for the present study. The distribution of the transverse shear stresses $\bar{\tau}_{y z}$ and $\bar{\tau}_{x z}$ across thickness is shown in Figure 11. It can be noted that these stresses not parabolic like the homogeneous plate. Unlike the in-plane 

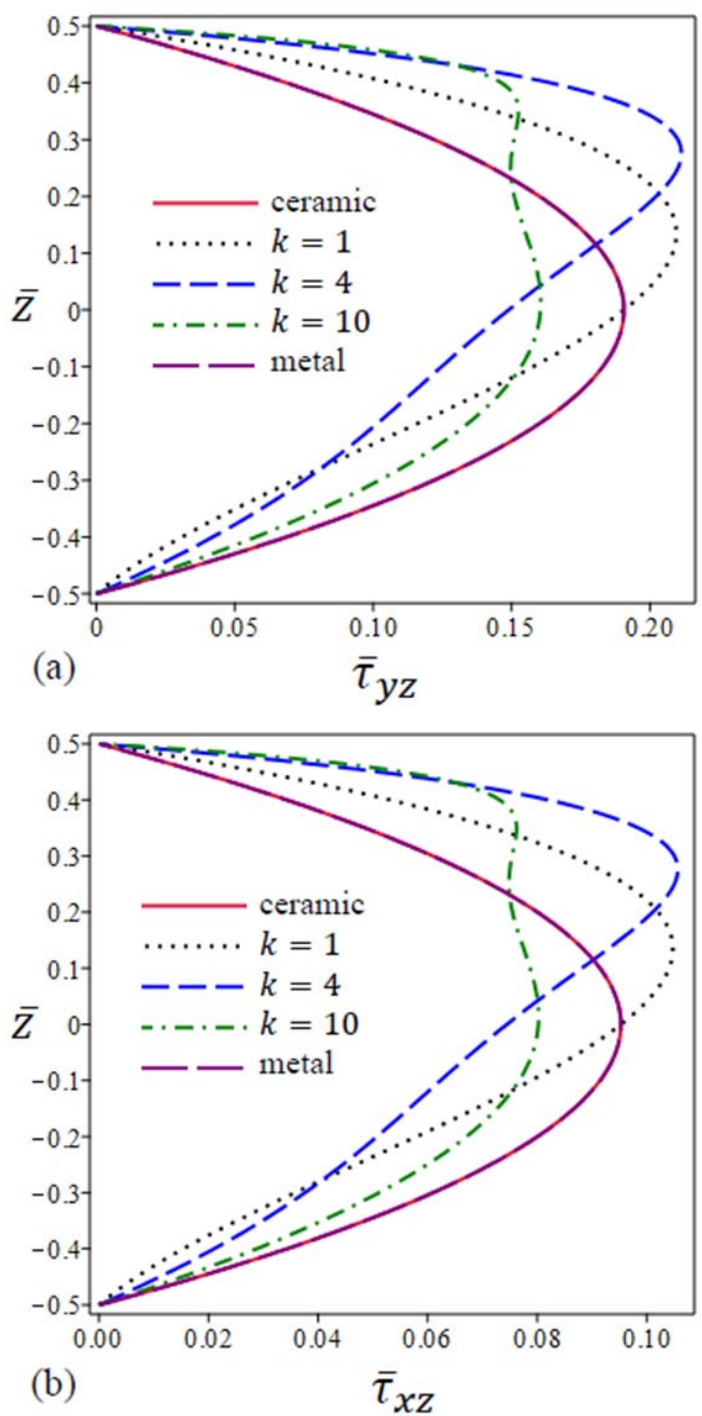

Figure 11: The transverse shear stresses across the thickness of a FG plate: (a) $\bar{\tau}_{y z}$, (b) $\bar{\tau}_{x z}$.

axial stresses $\bar{\sigma}_{x}$ and $\bar{\sigma}_{y}$, the in-plane tangential stress $\bar{\tau}_{x y}$ becomes tensile at the lower surface and compress at the upper surface and the homogeneous plate gives the maximum tensile stress at the lower surface and the minimum compressive stress at the upper surface of the FG rectangular plate as shown in Figure 12.

\section{Conclusions}

A refined quasi-3D shear and normal deformation plate theory for analyzing bending of FG plate with opposite simply-supported edges is introduced. The present theory consider the influence of the transverse normal strains not

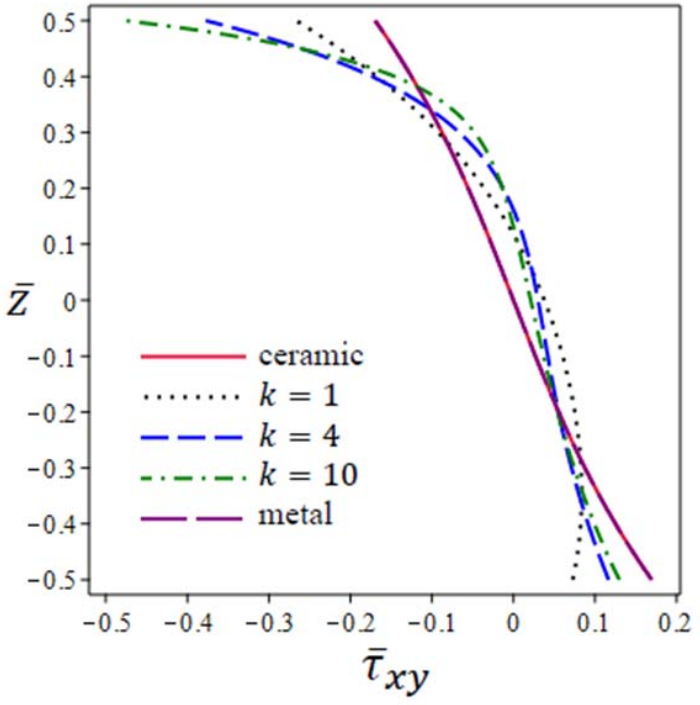

Figure 12: The in-plane tangential stress across the thickness of a FG plate.

like other shear deformation theories that ignored $\varepsilon_{z}$. The virtual work principle is used to obtain the governing equilibrium equations. Double Fourier series are used to minimize the equilibrium equations to a collection of coupled ordinary differential equations with variable coefficients. The accuracy of the present theory is established by comparisons with published results. From the discussion of figures and tables, we summarize the following conclusions:

- The present refined quasi-3D shear and normal deformation theory shows excellent agreement with the available results of different shear and normal deformation theories.

- The results clarify that the shear deformation theory $\left(\varepsilon_{z}=0\right)$ and the refined quasi-3D shear and normal deformation theory $\left(\varepsilon_{z} \neq 0\right)$ have very close results for the case of thin plates.

- For the case of thick plates and moderately thick plates it can be observed that the refined quasi-3D shear and normal deformation theory that cares for the transverse normal strain influence $\left(\varepsilon_{z} \neq 0\right)$, gives better results when compared to other theories that ignore the thickness stretching impact.

- The influences of transverse normal strain on the bending of FG plates should be taken into consideration. 


\section{References}

[1] M. Yamanouchi, M. Koizumi, T. Hirai, I. Shiota, Sendai, Japan, (1990).

[2] X. Zhao, K.M. Liew, Comput. Methods. Appl. Mech. Eng. 198 2796-2811 (2009).

[3] S. Hosseini-Hashemi, H.R.D. Taher, H. Akhavan, M. Omidi, Appl. Math. Model. 34 1276-1291 (2010).

[4] H.T. Thai, D.H. Choi, Finite. Elem. Anal. Des. 75 50-61 (2013).

[5] Z.Q. Cheng, R.C. Batra, Arch. Mech. 52 143-158 (2000).

[6] E. Carrera, S. Brischetto, M. Cinefra, M. Soave, Mech. Adv. Mater. Struct. 17 603-621 (2010)

[7] S. Vel, R. Batra, AIAA. J. 40 1421-1433 (2012).

[8] J.N. Reddy, Int. J. Numer. Meth. Eng. 684 663-684 (2000).

[9] A.M. Zenkour, Int. J. Solids. Struct. 42 5224-5242 (2005).

[10] M. Bouazza, Y. Kenouza, N. Benseddiq, A.M. Zenkour, Compos. Struct. 182 533-541 (2017).

[11] H.T. Thai, D.H. Choi, Compos. Struct. $101332-340$ (2013).

[12] H.M. Navazi, H. Haddadpour, M. Rasekh, Thin-Walled. Struct. 44 1129-1137 (2006).

[13] A. Rezaei Mojdehi, A. Darvizeh, A. Basti, H. Rajabi, Eng. Anal. Boundary. Elem. 35 1168-1180 (2011).

[14] J. Kim, J.N. Reddy, Compos. Struct. 103 86-98 (2013).

[15] W. Liu, Z. Zhong, Tsinghua. Sci. Technol. 14 58-63 (2009).

[16] H. Matsunaga, Compos. Struct. 87 344-357 (2009).

[17] H. Zhang, J-K. Jiang, Z-C. Zhang, Appl. Math. Mech. 35 467-478 (2014).

[18] K. Swaminathan, D.T. Naveenkumar, A.M. Zenkour, E. Carrera, Compos. Struct. 120 10-31 (2015).

[19] J.L. Mantari, C.G. Soares, Int. J. Mech. Sci. 78 60-71 (2014).

[20] A. Benachour, H.D. Tahara, H.A. Atmanea, A. Tounsia, M.S Ahmed, Compos. Part B. Eng. 42 1386-1394 (2011).

[21] A.M. Zenkour, Int. J. Appl. Mech. 5 (2013), 1350020 (15 pages) June.

[22] E. Carrera, S. Brischetto, M. Cinefra, M. Soave, Compos. B 42 123-33 (2011).

[23] A.M.A. Neves, A.J.M. Ferreira, E. Carrera, C.M.C. Roque, M. Cinefra, R.M.N. Jorge, C.M.M. Soares, Mech. Res. Commun. $38368-$ 371 (2011)
[24] A.M. Zenkour, Appl. Math. Model 37 9041-9051 (2013).

[25] A.M. Zenkour, Appl. Math. Model. 30 67-84 (2006).

[26] L. Dongdong, D. Zongbai, X. Huaizhi, Compos. Part B 106 107119 (2016).

[27] S. Akavci, Composites Part B 96 136-152 (2016).

[28] A.M. Zenkour, M.N.M. Allam and A.F. Radwan, Int. J. Appl. Mech. 61450063 (26 pages) (2014).

[29] A.M. Zenkour, A.F. Radwan, J. Sandw. Struct. Mater., 20 169-190 (2018).

[30] A.M. Zenkour, A.F. Radwan, Arch. Civil Mech. Eng. 18, 645-658 (2018).

[31] L. Dongdong, D. Zongbai, C. Guoping, X. Huaizhi, Z. Lujia, Compos. Struct. 169 29-41 (2017).

[32] J. Rouzegar, A. Abbasi, Thin. Walled. Struct. 120 386-396 (2017).

[33] A.M.A. Neves, A.J.M. Ferreira, E. Carrera, C.M.C. Roque, M. Cinefra, R.M.N. Jorge, C.M.M. Soares, Compos. Struct. 94 1814-1825 (2012).

[34] A.M.A. Neves, A.J.M. Ferreira, E. Carrera, C.M.C. Roque, M. Cinefra, R.M.N. Jorge, C.M.M. Soares, Compos. Part B 43 711-725 (2012).

[35] M. Talha, B.N. Singh, Appl. Math. Model. 34 3991-4011 (2010).

[36] J.N. Reddy, Int. J. Aerosp. Lightweight Struct. 11-21 (2011).

[37] J.L. Mantari, C.G. Soares, Compos. Struct. 94 2561-2575 (2012).

[38] H-T. Thai, K. Seung-Eock, Compos. Struct. 99 172-180 (2013).

[39] J.L. Mantari, C.G. Soares, Compos. Struct. 109 231-239 (2014).

[40] J.L. Mantari, C.G. Soares, Acta Mech. 226 625-642 (2015).

[41] S.S. Akavci, A.H. Tanrikulu, Compos. Part B 83 203-215 (2015).

[42] G. Bao, L. Wang, Int. J. Solids. Struct. 32 2853-2871 (1995).

[43] T. Mori, K. Tanaka, Acta Metall. 21571-574 (1973).

[44] F. Delale, F. Erdogan, ASME. J. Appl. Mech. 50 609-614 (1983).

[45] R.P. Shimpi, H.G. Patel, Int. J. Solids Struct. 43 6783-6799 (2006).

[46] A.M. Zenkour, J. Sandw. Struct. Mater. 15 629-656 (2013).

[47] A.M. Zenkour, AIAA J. 52 1466-1473 (2014).

[48] A.M. Zenkour, Compos. Struct. 122 260-270 (2015).

[49] A.M. Zenkour, Sadhana Acad. P. Eng. S. 40 215-234 (2015).

[50] H.T. Thai, S.E. Kim, Compos. Struct. 99 172-80 (2013).

[51] H.T. Thai, S.E. Kim, Int. J. Mech. Sci. 54 269-276 (2012). 